'Departamento de Medicina Familiar.

${ }^{2}$ Departamento de Salud Pública.

Unidad de Medicina

Basada en Evidencia.

Facultad de Medicina

Pontificia Universidad

Católica de Chile. Santiago de Chile.

Correspondencia a: Dra. Solange Rivera. E-mail: strivera@uc.cl

\section{Análisis crítico de un artículo: terapia de reemplazo de la nicotina, efectividad en un escenario real}

\author{
SOLANGE RIVERA M..$^{1,3}$, CLAUDIA BAMBS S. ${ }^{2}$ \\ Critically appraised article
}

\begin{abstract}
Objective: To examine the population effectiveness of nicotine replacement therapies (NRTs), either with or without professional counselling, and provide evidence needed to better inform healthcare coverage decisions. Methods: A prospective cohort study was conducted in three waves on a probability sample of 787 Massachusetts adult smokers who had recently quit smoking. The baseline response rate was 46\%; follow-up was completed by $56 \%$ of the designated cohort at wave 2 and $68 \%$ at wave 3. The relationship between relapse to smoking at follow-up interviews and assistance used, including NRT with or without professional help, was examined. Results: Almost one-third of recent quitters at each wave reported to have relapsed by the subsequent interview. Odds of relapse were unaffected by use of NRT for $>6$ weeks either with $(p=0.117)$ or without $(p=0.159)$ professional counseling and were highest among prior heavily dependent persons who reported NRT use for any length of time without professional counselling (OR 2.68). Conclusions: This study finds that persons who have quit smoking relapsed at equivalent rates, whether or not they used NRT to help them in their quit attempts. Cessation medication policy should be made in the larger context of public health, and increasing individual treatment coverage should not be at the expense of population evidence-based programmes and policies.
\end{abstract}

\section{Conclusión de los revisores}

En el largo plazo y a nivel poblacional las terapias de reemplazo de nicotina (TRN), específicamente parches y chicles de nicotina, podrían no ser efectivas para la mantención del cese del hábito tabáquico, en especial en grupos de pacientes con alto riesgo de recaídas. Si bien esta evidencia no parece ser suficiente para cambiar la conducta clínica actual en relación al manejo del tabaquismo, enfatiza la necesidad de explorar intervenciones adicionales para lograr este objetivo.

\section{Pregunta clínica}

En adultos que dejaron de fumar recientemente, ¿cuál es la efectividad de las terapias de reemplazo de nicotina, asociadas o no a consejería profesional, para evitar las recaídas?

\section{Introducción}

Según la última Encuesta Nacional de Salud, la prevalencia actual de tabaquismo en Chile es de 40,6\% entre las personas mayores de 15 años de edad $^{1}$. El manejo del hábito tabáquico a nivel in- 
Terapia de reemplazo de la nicotina - S. Rivera et al

dividual y poblacional es complejo, ya que se trata de una sustancia legal cuyo uso está fuertemente arraigado en diversas culturas, con implicancia en la actividad económica de distintos países ${ }^{2}$. El consumo de tabaco se relaciona, entre otras causas, con la propiedad adictiva de la nicotina; ésta es capaz de producir dependencia y síndrome de privación ${ }^{3}$. Los medicamentos más estudiados y usados para el manejo de la dependencia al tabaco son aquellos que contienen nicotina. Su efectividad ha sido probada a través de múltiples estudios aleatorizados ${ }^{4}$, sin embargo, la generalización de estos resultados a estudios poblacionales ha sido controvertida, ya que estos últimos han demostrado resultados opuestos ${ }^{5,6}$. El siguiente análisis crítico revisará un estudio observacional que generó interés en algunos medios de comunicación durante el año 2012.

\section{Características del estudio}

Tipo de estudio: Estudio de cohorte concurrente, realizado en tres fases (2001-2, 2003-4 y 2005-6). Los sujetos analizados fueron aquellos que en la fase 1 ó 2 reportaron haber dejado de fumar durante los dos años previos y que completaron una entrevista en la fase siguiente. Un total de 787 adultos habían dejado de fumar durante los dos años previos a la primera fase; de ellos 480 completaron la entrevista en la segunda fase, siendo la tasa de recaída $30,6 \%$. Por su parte, 364 adultos habían dejado de fumar durante los dos años previos a la segunda fase; de ellos, 248 completaron la entrevista en la tercera fase, siendo la tasa de recaída 31,6\%. Durante el seguimiento de los pacientes se evaluó la asociación entre recaída y la ayuda utilizada para dejar de fumar, incluyendo el uso de terapia de reemplazo de nicotina, con o sin consejería profesional. Otras variables medidas fueron: duración de la abstinencia reportada por los pacientes, grado de dependencia a la nicotina, género, edad, raza y nivel educacional.

\section{Validez (riesgo de sesgo) del estudio}

$\begin{array}{lc}\text { 1. ¿Los factores pronósticos que se sabe están } & \text { Parcial- } \\ \text { asociados con el resultado (outcome), eran si- } & \text { mente } \\ \text { milares en los distintos grupos de participantes } & \\ \text { en el estudio? } & \\ \begin{array}{l}\text { 2. ¿Fueron similares las circunstancias y métodos } \\ \text { utilizados para detectar los outcomes? }\end{array} & \text { Sí } \\ \text { 3. ¿El seguimiento fue suficientemente completo? } & \text { No }\end{array}$

\section{Resultados}

Ver Tablas 1 y 2.

Tabla 1. Proporción de sujetos que presentaron recaída, según fase y terapia recibida

\begin{tabular}{|c|c|c|c|c|}
\hline Terapia & $\begin{array}{l}\text { Proporción de recaí- } \\
\text { da entre fases } 1 \text { y } 2\end{array}$ & $\begin{array}{l}\text { Diferencia de } \\
\text { riesgo }\end{array}$ & $\begin{array}{l}\text { Proporción de recaí- } \\
\text { da entre fases } 2 \text { y } 3\end{array}$ & $\begin{array}{c}\text { Diferencia de } \\
\text { riesgo }\end{array}$ \\
\hline Sin terapia & $24 \%$ & $\begin{array}{l}\text { Categoría de } \\
\text { Referencia }\end{array}$ & $22 \%$ & $\begin{array}{l}\text { Categoría de } \\
\text { Referencia }\end{array}$ \\
\hline TRN & $34 \%$ & $\begin{array}{l}10 \text { recaídas adicionales } \\
\text { por cada } 100 \text { tratados }\end{array}$ & $52 \%$ & $\begin{array}{l}30 \text { recaídas adicionales } \\
\text { por cada } 100 \text { tratados }\end{array}$ \\
\hline $\begin{array}{l}\text { TRN y consejería } \\
\text { profesional }\end{array}$ & $25 \%$ & $\begin{array}{l}1 \text { recaída adicional por } \\
\text { cada } 100 \text { tratados }\end{array}$ & $30 \%$ & $\begin{array}{l}8 \text { recaídas adicionales } \\
\text { por cada } 100 \text { tratados }\end{array}$ \\
\hline
\end{tabular}

TRN: terapia de reemplazo a la nicotina.

Tabla 2. Odds Ratio de recaída en sujetos que habían dejado de fumar hace dos o más años, según grado de dependencia previa y terapia recibida

\begin{tabular}{|ccc|}
\hline Terapia & $\begin{array}{c}\text { Grado de dependencia previa: fuerte } \\
\text { Odds Ratio* (IC 95\%) }\end{array}$ & $\begin{array}{c}\text { Grado de dependencia previa: leve } \\
\text { Odds Ratio* (IC 95\%) }\end{array}$ \\
Sin terapia & 1,00 & 1,00 \\
TRN & - & - \\
& 2,68 & 1,38 \\
TRN y consejería & $(1,40-5,11)$ & $(0,34-5,67)$ \\
profesional & 0,94 & 2,08 \\
\hline
\end{tabular}

TRN: terapia de reemplazo a la nicotina. IC 95\%: Intervalo de confianza 95\%. *Ajustados por duración de la abstinencia y edad. 


\section{Comentarios}

\section{Comentarios acerca de la validez \\ (riesgo de sesgo)}

Se trata de un estudio de cohorte concurrente de calidad moderada, que cumple parcialmente con algunos criterios de riesgo de sesgo. Por su naturaleza observacional, es altamente probable que las características basales de los sujetos que recibieron diferentes modalidades terapéuticas hayan diferido. En relación a otros factores pronósticos asociados a la ocurrencia del outcome, el estudio no reporta la dosis de terapia de reemplazo de nicotina utilizada ni el uso concomitante de otros fármacos que tengan alguna efectividad en el cese del tabaquismo o la prevención de recaídas. Otras variables conocidas por su valor pronóstico en cesación de tabaquismo, como nivel de motivación, soporte social, trastornos del ánimo y dependencia concomitante a alcohol o drogas ilícitas, tampoco fueron incluidas en el estudio. Por lo anterior, es altamente probable que los resultados del estudio estén afectados por el sesgo de confusión. La metodología utilizada para recoger información y detectar el outcome parece adecuada para este diseño. Una de las principales amenazas a la validez del estudio es la pérdida de seguimiento, hecho que introduce la posibilidad de sesgo debido a pérdidas diferenciales.

\section{Comentarios acerca de los resultados}

Aproximadamente un tercio de los ex-fumadores declaró haber recaído: 30,6\% y 31,3\% en la segunda y tercera fase, respectivamente. El único predictor de recaída entre las fases 1 y 2 fue la duración del tiempo de abstinencia: $35 \%$ en quienes reportaron abstinencia durante menos de 6 meses vs $17 \%$ entre quienes reportaron abstinencia por más de 6 meses $(p<0,001)$. Por su parte, entre las fases 2 y 3 el tipo de TRN utilizado fue predictor de recaída: TRN sin consejería profesional (52\%), TRN con consejería profesional (30\%) y ninguna terapia $(22 \%)(\mathrm{p}=0,015)$.

En el subgrupo de sujetos con grado de dependencia previa fuerte, la posibilidad de recaída fue mayor en aquellos que reportaron uso de TRN sin consejería profesional, comparado con quienes no utilizaron terapia alguna, siendo el OR $=2,68$ (IC95\% 1,40-5,11). El uso de Odds Ratio como medida de efecto en este estudio (cohorte concurrente, temporalmente prospec- tiva) se fundamenta en la necesidad de utilizar modelos matemáticos para ajustar los resultados por variables consideradas confundentes. No cabe duda que OR es la medida de riesgo apropiada para expresar los resultados en este estudio. Lamentablemente, la mayor desventaja del OR es su difícil interpretación clínica. Una lectura más detallada del OR sería la siguiente: en el subgrupo de sujetos con grado de dependencia previa fuerte, la razón recaída/no-recaída de quienes recibieron TRN sin consejería profesional fue 2,68 veces la razón recaída/no-recaída de quienes no recibieron terapia alguna. Otras formas de expresar este OR en palabras son:

- Los pacientes que recibieron la intervención tuvieron una chance (odds) de recaída 2,68 veces la del grupo control.

- La intervención incrementó la chance (odds) a $268 \%$ de la chance (odds) en el grupo control. - La intervención incrementó las chances (odds) de recaída en $168 \%$.

El tamaño del efecto fluctuó entre 1,40 y 5,11 (límites inferior y superior del intervalo de confianza 95\% para el OR). Este intervalo de confianza no incluye el valor del "no efecto" (es decir, OR $=1,00)$.

\section{Comentarios acerca de la aplicabilidad}

El cese del hábito tabáquico es el principal outcome a lograr con intervenciones que ayuden a dejar de fumar; sin embargo, 35 a $40 \%$ de los pacientes suelen recaer a los 5 años de suspender el tabaco ${ }^{7}$. En este escenario adquiere suma importancia la identificación de factores asociados a la prevención de las recaídas. La población del estudio es una muestra de ex-fumadores, siendo el outcome "recaída" clínicamente relevante y medido en un plazo razonable ( 2 años). Sin embargo, no se midieron outcomes tales como efectos adversos asociados al uso de TRN.

$\mathrm{Si}$ bien se midieron algunas variables que pudieran asociarse con mayor o menor probabilidad de presentar recaída ${ }^{8,9}$ (tiempo de uso de la TRN, grado de dependencia a la nicotina previo al cese, edad, género, raza y nivel educacional), el modelo explicativo de recaídas es sin duda de mayor complejidad y algunos factores de importancia no fueron incluidos (presencia de trastornos del ánimo y otras adicciones, estrés, apoyo social y niveles de motivación para dejar de fumar ${ }^{10,11}$ y no 
Terapia de reemplazo de la nicotina - S. Rivera et al

se explicita la forma en que se midió dependencia. Otro aspecto relacionado a la aplicabilidad de esta intervención tiene que ver con el tiempo de uso de la TRN: sólo un tercio de los usuarios en las fases 1 y 2 la utilizó durante el período recomendado (8 semanas).

Finalmente, es importante recordar que cuando el objetivo es medir la eficacia de una intervención terapéutica, el diseño con menor riesgo de sesgo es el Ensayo Clínico Aleatorizado (ECA). Si, además, quisiéramos tomar decisiones en base a la totalidad de la evidencia existente, las revisiones sistemáticas de ECA proporcionan esta información con riesgo de sesgo aún menor. Una de las dificultades que tienen los ECA tiene relación con su capacidad de medir la efectividad de una intervención (es decir, qué tan útil es la intervención en un escenario "real"). Es en este punto donde el estudio analizado constituye un aporte, ya que se mide lo que los investigadores llaman la "efectividad en el mundo real" de la TRN (por ejemplo, esta encuesta fue aplicada sin excluir a los fumadores con altos niveles de dependencia). En Chile, cerca de un tercio de los fumadores tienen un alto nivel de dependencia, de modo que incorporar este tipo de población se interpreta como una fortaleza en términos de aplicabilidad. En la misma línea, es difícil encontrar ensayos clínicos aleatorizados que evalúen efectividad en términos de recaída en plazos mayores a los 12 meses $^{7}$.

Contrastando los resultados obtenidos, existen revisiones sistemáticas que muestran efectividad de la TRN para la prevención de recaídas ${ }^{7,12}$, por lo que a nivel individual siguen siendo una alternativa farmacológica para el manejo del tabaquismo, especialmente si su uso se realiza en las dosis y combinaciones adecuadas para cada caso en particular. De todos modos, el estudio analizado constituye evidencia que viene a complementar el conocimiento derivado de los ECA en el tema de la efectividad de las terapias para dejar de fumar. Sin embargo, en ningún caso se puede generalizar su resultado. Lo que sí rescatamos del estudio analizado es haber identificado un grupo con alto riesgo de recaída (por múltiples variables no medidas en este estudio), en quienes existe una brecha entre eficacia y efectividad. Este grupo es especialmente interesante para aplicar intervenciones adicionales, las cuales deben ser puestas a prueba en futuros estudios aleatorizados. Estos hallazgos pueden contribuir a la toma de decisiones sobre políticas públicas relacionadas con medidas para promover la cesación del tabaquismo.

\section{Referencias}

1. Ministerio de Salud. Encuesta Nacional de Salud 2009-2010. http://www.encuestasalud.cl/pdf/InformeENS_2009-2010_CAP5.pdf).

2. Evans N, Farkas A, Gilpin E, Berry C, Pierce JP. Influence of tobacco marketing and exposure to smokers on adolescent susceptibility to smoking. J Natl Cancer Inst 1995; 87: 1538-45.

3. Lee E, D'alonzo GE. Cigarette smoking, nicotine addiction, and its pharmacology treatment. Arch Intern Med 1993; 153: 34-48.

4. Stead LF, Perera R, Bullen C, Mant D, Lancaster T. Nicotine replacement therapy for smoking cessation. Cochrane Database Syst Rev 2008; (1): CD000146.

5. Fiore MC, Novotny TE, Pierce JP, Giovino GA, Hatziandreu EJ, Newcomb PA, et al. Methods used to quit smoking in the United States. Do cessation programs help? JAMA. 1990 May 23-30; 263 (20): 2760-5. Erratum in: JAMA 1991; 265 (3): 358.

6. Chapman $\mathrm{S}$. The inverse impact law of smoking cessation. Lancet 2009; 373: 701e3.

7. Hajek P, Stead LF, West R, Jarvis M, Lancaster T. Relapse prevention interventions for smoking cessation. Cochrane Database Syst Rev. 2009; (1): CD003999.

8. Norregaard J, Tonnesen P, Petersen L. Predictors and reasons for relapse in smoking cessation with nicotine and placebo patches. Prev Med 1993; 22 (2): 261-71.

9. Kenford SL, Fiore MC, Jorenby DE, Smith SS, Wetter D, Baker TB.Predicting smoking cessation. Who will quit with and without the nicotine patch. JAMA 1994; 271 (8): 589-94.

10. Quesada M, Carreras JM, Sánchez L. Recaída en el abandono del consumo de tabaco: una revisión. Adicciones, 2002; 14 (1): 65-78.

11. Lawhon D, Humfleet GL, Hall SM, Muñoz RF, Reus VI. Longitudinal analysis of abstinence-specific social support and smoking cessation. Health Psychol 2009; 28 (4): 465-72.

12. Agboola S, Mcneill A, Coleman T, Leonardi Bee J. A systematic review of the effectiveness of smoking relapse prevention interventions for abstinent smokers. Addiction 2010; 105 (8): 1362. 